\title{
Overexpression of Hedgehog signaling molecules and its involvement in triple-negative breast cancer
}

\author{
YAJUN TAO ${ }^{1}$, JUN MAO ${ }^{2}$, QINGQING ZHANG $^{2}$ and LIANHONG LI ${ }^{2}$ \\ ${ }^{1}$ Department of Pathology, Medical College of Dalian University, Dalian, Liaoning 116622; \\ ${ }^{2}$ Department of Pathology, Dalian Medical University, Dalian, Liaoning 116044, P.R. China
}

Received March 29, 2011; Accepted July 1, 2011

DOI: $10.3892 / \mathrm{ol} .2011 .357$

\begin{abstract}
The purpose of this study was to investigate the activation of Hedgehog (Hh) signaling molecules and its involvement in triple-negative breast cancer (TNBC). A total of 123 cases of paraffin blocks, including 83 cases of primary breast carcinoma, 30 cases of mammary hyperplasia and 10 cases of normal breast tissue, were immunohistochemically analyzed for Sonic Hedgehog (SHH), Patched-1 (PTCH1), Smoothened (SMO) and glioma-associated oncogene homoglog 1 (GLI1) expression. The expression of SMO and GLI1 in TNBC was significantly increased in comparison to non-triple-negative breast cancer (nTNBC). GLI1 expression manifested an inverse association with the estrogen receptor. The levels of GLI1 expression were increased in lymph nodepositive cases. The expression of SHH and SMO was increased in high histological grades. Furthermore, the expression of SMO and GLI1 was correlated with superior tumor stage. The expression of SHH, SMO and GLI1 was significantly increased in breast cancer and mammary hyperplasia. PTCH1 expression was significantly decreased in breast cancer compared to mammary hyperplasia and normal breast tissue. For the first time, clinical evidence has been provided in support of significant roles of Hh signaling in TNBC. Hh signaling is involved in breast ductal changes and malignant transformation. Measures to inhibit Hh activity may improve the prognosis of TNBC patients.
\end{abstract}

\section{Introduction}

Breast cancer is a heterogeneous disease. Gene expression analysis has identified molecular classes of breast cancer that are biologically and clinically distinct (1). One of these

Correspondence to: Dr Lianhong Li, Department of Pathology, Dalian Medical University, 9 Western Section, Lvshun South Street, Lvshunkou, Dalian, Liaoning 116044, P.R. China

E-mail: lilianhong@dlmedu.edu.cn

Key words: Sonic Hedgehog, Patched-1, Smoothened, gliomaassociated oncogene homoglog 1, triple-negative breast cancer, cancer stem cell subgroups, which has attracted significant attention in recent years, is triple-negative breast cancer (TNBC). TNBC is defined as a group of tumors that neither expressing estrogen receptors (ER) and progesterone receptors (PR) nor overexpressing HER2. Studies have revealed that TNBC accounts for $10-17 \%$ of all breast carcinomas $(2,3)$ and is more aggressive in that considerable recurrence and metastasis occurs (4-7), and relatively few patients exhibit favorable survival times $(3,8,9)$. Due to reduced response to hormonal therapy and poor prognosis $(10,11)$, new targeted therapies for TNBC are required to ameliorate breast cancer treatment.

The Hedgehog (Hh) signaling pathway involves a signaltransduction cascade that includes a member of the Hedgehog family of secreted ligands [Sonic Hedgehog (SHH), Indian Hedgehog ( $\mathrm{IHH})$ or Desert Hedgehog (DHH)] in mammals. $\mathrm{Hh}$ binds one or more Patched family hedgehog receptors [Patched-1 (PTCH1) and Patched-2 (PTCH2)]. In the absence of ligand, PTCH inhibits downstream signaling by limiting the function of the Smoothened (SMO), a seven-transmembrane protein with strong resemblance to a G-protein-coupled receptor. In the presence of ligand, $\mathrm{PTCH}$ releases inhibition of SMO, leading to induction of the target transcription factors glioma-associated oncogene homoglog (GLI1, GLI2 or GLI3) (12). Dysregulation of Hh signaling has been shown to induce malformation of the organism and carcinogenesis in experimental systems (13-16). Previous studies emphasized the importance of a tightly controlled Hh pathway activation in the mammary gland to ensure proper development and avert tumor formation $(17,18)$. The exact role of activated $\mathrm{Hh}$ signaling in the development or progression of tumors remains to be elucidated. However, certain studies have indicated that Hh signaling plays a role in stem/progenitor cell maintenance and self-renewal, which is regarded as a significant marker of cancer stem cell (CSC) activation $(19,20)$. Nevertheless, the role of this pathway in breast CSCs has yet to be determined.

In the present study, we addressed other types of breast cancer that exhibit expression of ER, PR or overexpression of HER2 as non-triple-negative breast cancer (nTNBC). The primary purpose of this study was, first to explore the activation of $\mathrm{Hh}$ in TNBC by analyzing the expression difference of Hh signaling between TNBC and nTNBC; and second, to elucidate the relationship between $\mathrm{Hh}$ molecules and clinicopathological parameters to gain a better understanding of the Hh pathway in TNBC. In addition, expression of $\mathrm{Hh}$ 
molecules in breast cancer, mammary hyperplasia and normal breast tissue was analyzed to study the role of the Hh pathway in breast ductal changes.

\section{Materials and methods}

Clinical samples. In total, 123 cases of paraffin blocks, including 83 cases of primary breast carcinoma, 30 cases of mammary hyperplasia and 10 cases of normal breast tissue, were selected from the Department of Pathology, Dalian Central Hospital (Dalian, China), between 2007 and 2010, following approval of the study by the Institutional Human Ethics Committee. The normal breast tissues were obtained from the resection margins of modified radical mastectomy specimens of breast cancer. The ages of the patients ranged from 32 to 76 years (average 52.3). Immunopathology analysis according to the expression of ER, PR and HER2 contained in breast cancer revealed that there were $23 \mathrm{TNBC}$ specimens. In addition, of 83 cases of primary breast carcinoma, 73 invasive ductal carcinomas (IDC) and 10 invasive lobular carcinomas were confirmed by histopathological examination, of which the 23 TNBC specimens were IDC.

The clinicopathological parameters, including age, tumor size, differentiation and the presence of nodal metastasis, were evaluated. The $23 \mathrm{TNBC}$ specimens comprised 3 cases of pTNM stage I, 7 cases of pTNM stage IIa, 5 cases of pTNM stage IIb, 4 cases of pTNM stage IIIa and 4 cases of pTNM stage IV. TNM staging was assessed according to the staging system established by the American Joint Committee on Cancer (AJCC) (21).

Immunohistochemical staining. The sections were deparaffinized in xylene and rehydrated with graded ethanol followed by a microwave heating for $30 \mathrm{~min}$ in $10 \mathrm{mM}$ sodium citrate buffer ( $\mathrm{pH} 7.2) ; 0.3 \%$ hydrogen peroxide solution was used for the blocking of endogenous peroxidase activity. The primary monoclonal antibodies against SHH (ab53281; Abcam, Cambridge, UK), PTCH1 (BS1286; Bioworld, Atlanta, Georgia, USA), SMO (ab72130; Abcam), GLI1 (sc-20687; Santa Cruz Biotechnology, Santa Cruz, CA, USA), ER (M7047; Dako, Denmark), PR (M3569; Dako) and HER2 (A0485; Dako) were applied at $4^{\circ} \mathrm{C}$ overnight. The dilutions in PBS were as follows: SHH, 1:100; PTCH1, 1:50; SMO, 1:100; GLI1, 1:100; ER, 1:50; PR, 1:50 and HER2, 1:100. Sections were then incubated with HRP-labeled goat anti-mouse/rabbit antibody (Maixin Bio) for $30 \mathrm{~min}$ at room temperature. 3,3'-Diaminobenzidine was used as the chromogen and hematoxylin as the nuclear counterstain. The sections were dehydrated, cleared and mounted.

Immunoblot analysis. Tissue lysates were prepared from 1 TNBC and 1 nTNBC specimen. Frozen tissue samples were homogenized and lysed in RIPA buffer containing $1 \mathrm{X}$ protease inhibitor cocktail. Protein concentrations were determined using the Bradford assay. Equal amounts of proteins (80 $\mu \mathrm{g} /$ lane) from tissue lysates were electrophoresed in $10 \%$ sodium dodecyl sulfate-polyacrylamide gels. The proteins were then transferred onto polyvinylidenedifluoride membranes. After blotting in 5\% non-fat dry milk in Trisbuffered saline, blots were incubated with anti-SHH antibody
(1:500 dilution), anti-PTCH1 antibody (1:100 dilution), anti-SMO antibody (1:200 dilution) and anti-GLI1 antibody (1:200 dilution) at $4^{\circ} \mathrm{C}$ overnight. Membranes were incubated with a secondary antibody, HRP-labeled goat anti-mouse/ rabbit antibody, diluted at an appropriate dilution in 1\% BSA for $2 \mathrm{~h}$ at room temperature. Protein bands were visualized on X-ray film using an enhanced chemiluminescence system (Santa Cruz).

Evaluation of immunohistochemical staining. According to the scoring method of Sinicrope et al (22), the immuno-reactive score (IRS) was obtained by multiplication of staining intensity (graded between negative, 0 ; weak, 1; moderate, 2 and strong, 3) and the percentage of positively-stained cells (graded between 0 and 4 , where 0 , negative; $1,<25 \%$; $2,25-50 \% ; 3,51-75 \%$ and $4,>75 \%$ ). Cases with discrepancies in IRS were discussed together with two pathologists until consensus was reached. Final staining scores were represented as: negative (IRS 0), -; weak positive (IRS 1-4), +; moderate positive (IRS 5-8), ++; and strong positive (IRS 9-12), +++. Additionally, tumors with +++ scores were classified as overexpression for HER2, whereas tumors with -, + or ++ scores were considered as exhibiting no overexpression.

Statistical analysis. The data were statistically analyzed using the SPSS software version 13.0 (SPSS Inc., Chicago, IL, USA). Pearson's Chi-square test was performed to analyze the difference in expression of $\mathrm{H}$ molecules between TNBC and nTNBC, between breast cancer, mammary hyperplasia and normal breast tissue, and possible associations between $\mathrm{Hh}$ molecules and clinicopathological parameters. Spearman's rank correlation coefficients were calculated to analyze the associations between $\mathrm{Hh}$ molecules and ER, PR and HER2. Two-sided p-values of $<0.05$ were considered to be statistically significant.

\section{Results}

SHH, PTCHI, SMO and GLII expression in TNBC and $n T N B C$. SHH, PTCH1, SMO and GLI1 expression in TNBC and nTNBC are shown in Table I. Expression of SMO and GLI1 in TNBC was significantly increased in comparison to those in nTNBC ( $\mathrm{p}=0.006$ and $\mathrm{p}=0.022$, respectively). Furthermore, Western blot analysis independently confirmed the immunohistochemical findings of SHH, PTCH1, SMO and GLI1 protein expression in TNBC and paired nTNBC (Fig. 1). The relationship between SHH, PTCH1, SMO and GLI1 expression, and ER, PR and HER-2 expression was analyzed by Spearman's rank correlation coefficients (data not shown): the result revealed that GLI1 manifested an inverse association with ER ( $\mathrm{p}=0.015)$.

Relationships between the expression of SHH, PTCH1, SMO, GLII and clinicopathological parameters in TNBC. In the present study, we assessed clinicopathological characteristics, including age, lymphatic involvement, histologic grade and tumor stage (Table II). The level of GLI1 expression was increased in lymph node-positive cases $(\mathrm{p}=0.017)$. SHH and SMO expression was increased in specimens with high histological grades ( $\mathrm{p}=0.023$ and $\mathrm{p}=0.035$, respectively). The 


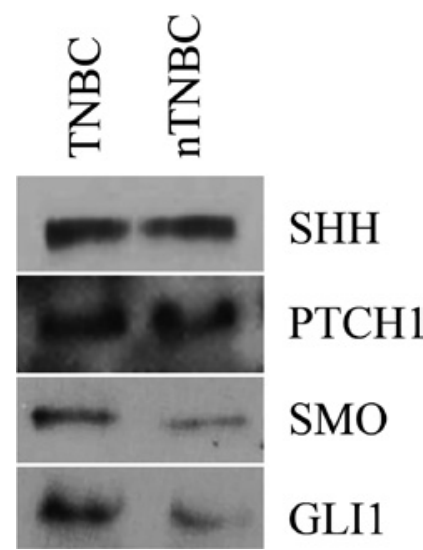

Figure 1. Analysis of the presence of SHH, PTCH1, SMO and GLI1 in TNBC and nTNBC. Western blotting was performed using anti-SHH, PTCH1, SMO and GLI1. The expression of SHH, PTCH1, SMO and GLI1 in TNBC and nTNBC is consistent with that detected by immunohistochemical staining.

expression of SMO and GLI1 was correlated with superior tumor stage ( $\mathrm{p}=0.042$ and $\mathrm{p}=0.032$, respectively).

SHH, PTCHI, SMO and GLII expression in breast cancer, mammary hyperplasia and normal breast tissue. The results of the immunohistochemical analysis of SHH, PTCH1, SMO and GLI1 expression in breast cancer, mammary hyperplasia and normal breast tissue are shown in Table III and Fig. 2. The expression of SHH was rare in normal breast epithelium, but was frequently observed in breast cancer and mammary hyperplasia ( $\mathrm{p}=0.000$ and $\mathrm{p}=0.000$, respectively). PTCH1 expression was significantly decreased in breast cancer compared to mammary hyperplasia and normal breast tissue. Significant differences were observed between breast cancer, mammary hyperplasia and normal breast tissue $(\mathrm{p}=0.000, \mathrm{p}=0.001$ and $\mathrm{p}=0.007$, respectively). Expression of SMO and GLI1 was completely absent in normal breast tissue, but was markedly increased in breast cancer and mammary hyperplasia (between breast cancer, mammary hyperplasia and normal breast tissue, differences of SMO expression were $\mathrm{p}=0.003, \mathrm{p}=0.012$ and $\mathrm{p}=0.000$, respectively; and differences of GLI1 expression were $\mathrm{p}=0.005, \mathrm{p}=0.02$ and $\mathrm{p}=0.000$, respectively).

\section{Discussion}

Hh signaling pathway is a highly conserved system for the regulation of patterning and growth in animal development. Tumorigenesis is concerned with abnormal activations in components of the Hh signaling pathway (23-26). The molecular mechanisms underlying this interaction remain unclear. However, using an in vitro suspension culture system for the breast CSCs, characterized by the cell surface phenotype $\mathrm{CD} 44^{+} \mathrm{CD} 24 /$ low and tumor initiating capacity, expression of SHH, Gli1 and Gli2 in these cells was observed at higher levels than in other types of breast cancer cells and was down-regulated when $\mathrm{CD} 44^{+} \mathrm{CD} 24$ /low cells were allowed to differentiate $(18,27)$. In concordance with this observation, activation of the Hh pathway by adding SHH protein or overexpressing GLI1, GLI2 or SmoM2 results in increased mammary mammosphere (MS) formation, which enriches stem cells (28). Conversely, inhibition of the Hh pathway 


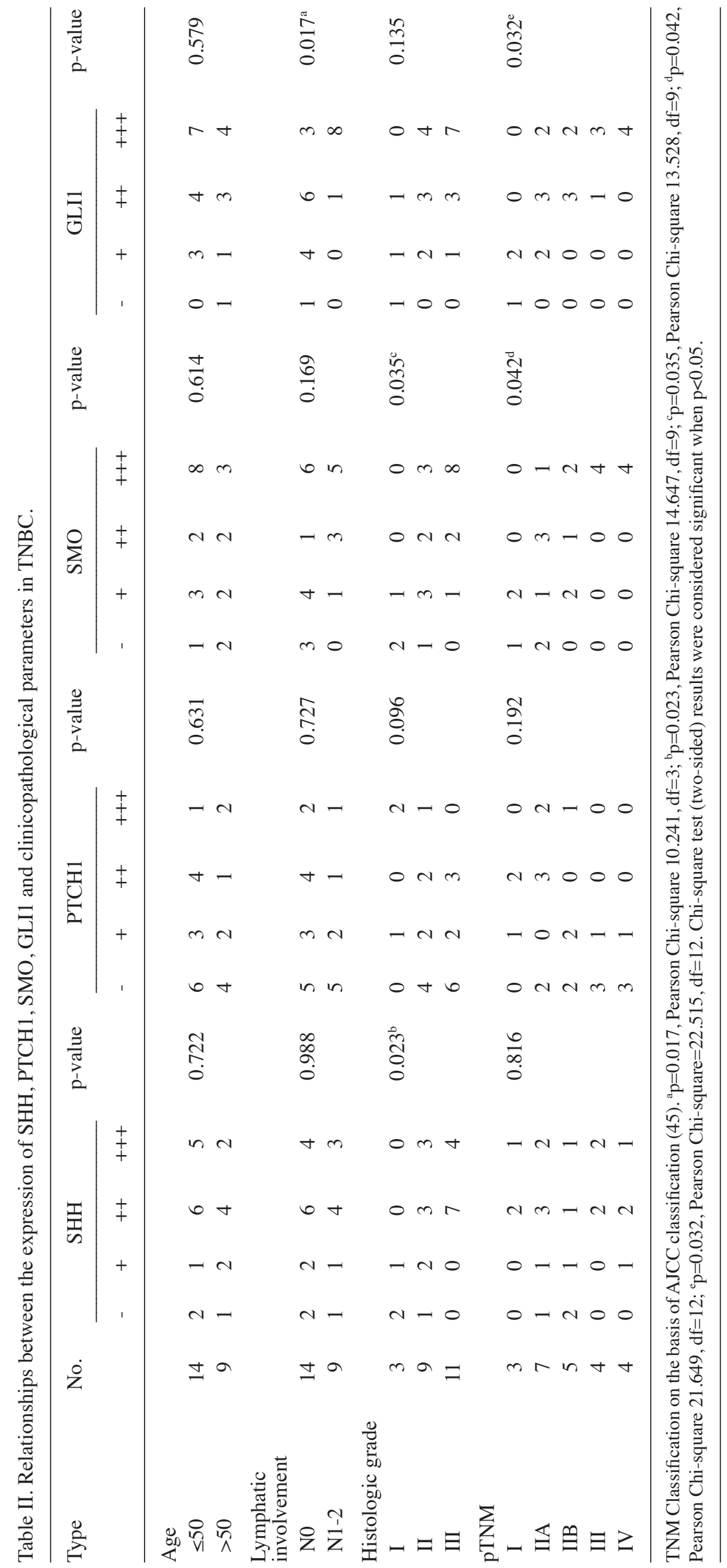




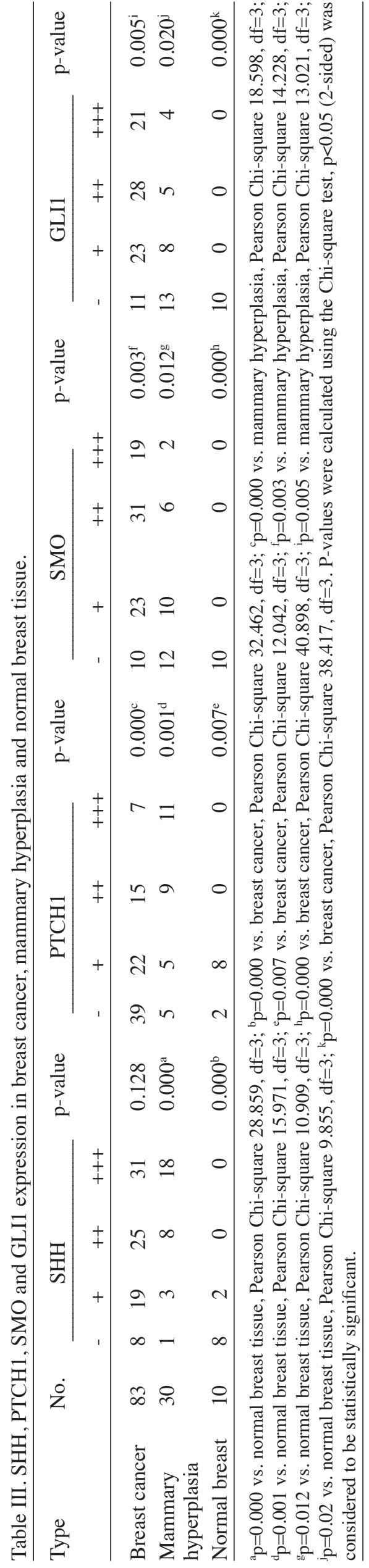

with cyclopamine was reported to decrease the MS formation efficacy of human mammary cells (20). These results indicate that the activation of $\mathrm{Hh}$ signaling plays an essential role in the maintenance of CSCs (29-31). With regard to TNBC with characteristics of cancer stem-like cells including more frequent recurrence, chemoresistance and shorter survival than nTNBC, we addressed the hypothesis that there are more active CSCs in TNBC compared to nTNBC, and Hh signaling may contribute to this activation. For the first time we provide clinical evidence to prove this hypothesis by analyzing the expression of SHH, PTCH1, SMO and GLI1 in TNBC. We noted that the key molecules of Hh signaling SMO and GLI1 expression in TNBC were markedly increased in comparison to nTNBC, indicating that Hh signaling in TNBC has been activated and contributes to the proliferative abilities of CSCs. Moreover, we observed that the expression of GLI1 was up-regulated in lymph node-positive cases, the expression of SHH and SMO was increased in high histological grade and the expression of SMO and GLI1 was correlated with superior tumor stage. These findings indicate that, by regulating the continuous activation of breast $\mathrm{CSCs}$, the Hh signaling pathway is involved, not only in progression, but also in invasion and metastasis. In addition, the expression of GLI1 was inversely correlated with ER, consistent with a previous study (32) that revealed that GII1 expression was decreased in ER-positive breast cancer cell lines MCF-7 and T47D. These data indicate that GLI1 may attenuate the response to estrogenic stimulation by negatively regulating ER signaling, which may be the reason for TNBC patients exhibiting insensitivity to hormone therapy. As noted, current systemic cancer therapies frequently fail to eradicate TNBC. Failure of these therapies to effectively target CSCs may account for this failure. Our results indicate that the development of agents that eliminate or control CSCs by inhibiting $\mathrm{Hh}$ signaling activation may be an effective strategy for TNBC prevention.

Hh signaling plays a role in breast ductal changes. It has been reported that targeted disruption of PTCH1 in mouse mammary stroma and expression of activated human Smo (SmoM2) under the MMTV promoter resulted in ductal dysplasia characterized by increased proliferation (28). To the best of our knowledge, expression of the Hh pathway in clinical mammary hyperplasia specimens has seldom been reported. Our clinical data revealed that SHH, SMO and GLI1 expression was increased and PTCH1 expression was decreased in mammary hyperplasia. The expression differences of SHH, PTCH1, SMO and GLI1 were observed between mammary hyperplasia and normal breast tissue. In addition, we observed that the expression of SMO and GLI1 was increased and PTCH1 expression was significantly decreased in breast cancer compared to mammary hyperplasia. These results indicate that $\mathrm{Hh}$ molecules were constitutively activated in the continuous process of normal breast tissuemammary hyperplasia-breast cancer, and the target of $\mathrm{Hh}$ molecules may be stem cells. Although breast stem cells are capable of self-renewal, they are relatively quiescent; in other words, these cells have proliferative capacity, but often do not cycle (33). Hh signaling may induce breast stem cells into the cell cycle to increase quantities of breast stem cells, thereby contributing to mammary hyperplasia formation. Furthermore, 

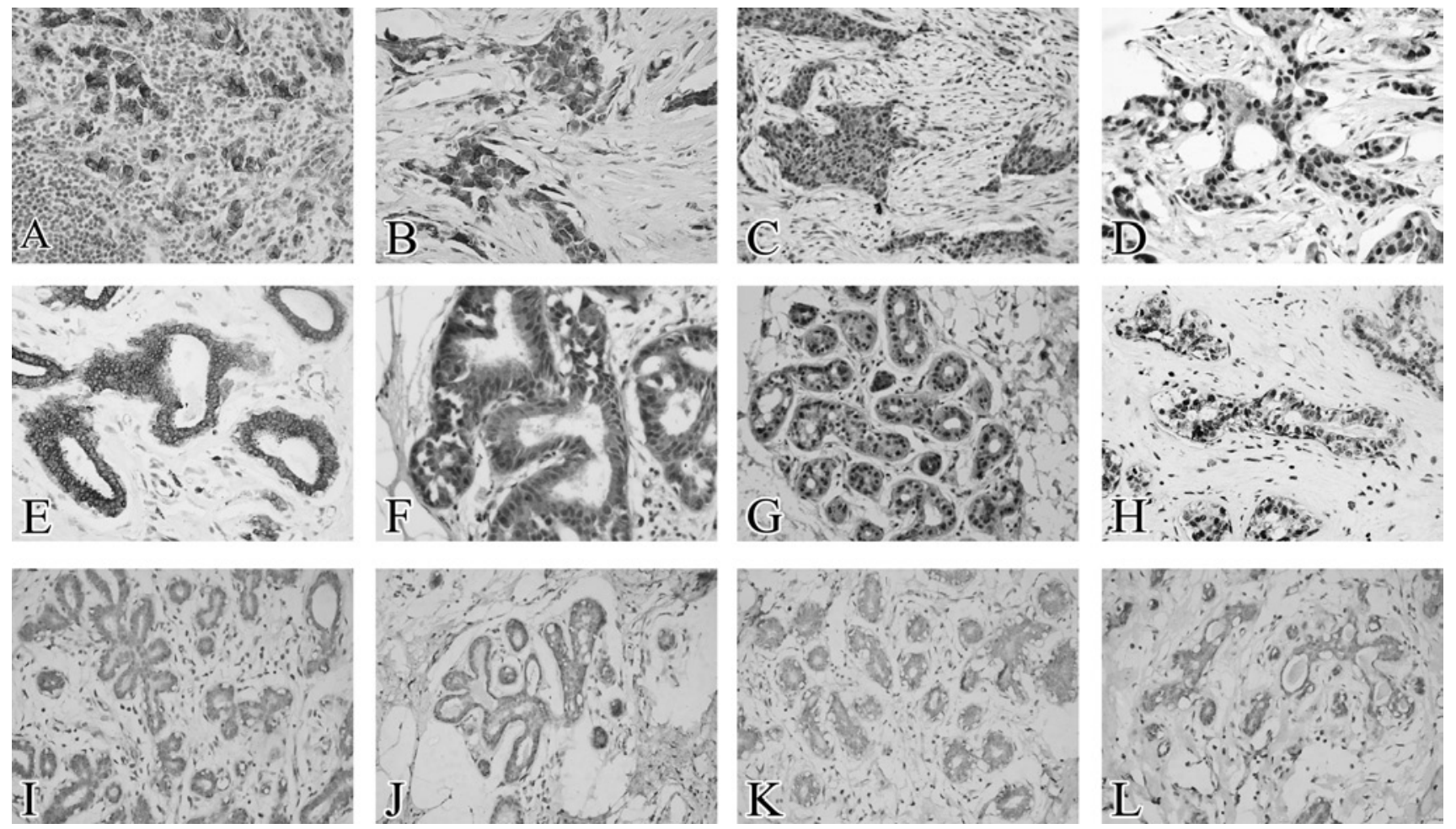

Figure 2. Expression of SHH, PTCH1, SMO and GLI1 in breast cancer, mammary hyperplasia and normal breast tissue. (A and E) SHH located in the membrane and cytoplasm of breast cancer and mammary hyperplasia. (B and F) PTCH1 mainly located in the cytoplasm of breast cancer and mammary hyperplasia. (C and G) SMO located in the cytoplasm and nucleus of breast cancer and mammary hyperplasia. (D and H) GLI1 mainly located in the nucleus of breast cancer and mammary hyperplasia. (I-L) Loss of SHH, SMO and GLI1 expression in normal breast tissue, respectively. (J) Weak positive expression of PTCH1 in normal breast tissue. Original magnification, $\mathrm{x} 400$.

the continuous activation or mutation of Hh signaling may cause breast stem cells to malignantly transform, resulting in oncogenesis and malignant progression. However, the mechanism of the Hh pathway in breast stem cell transformation requires further study.

In the present study, clinical evidence was provided in support of the crucial role of Hh signaling in TNBC. In addition, our clinical data also revealed that Hh signaling plays a role in breast ductal changes. We postulate that the potential mechanisms involved in the activation of stem cells, particularly the maintenance of malignant biological behaviour of breast CSCs and consequent promotion of tumor progression, are attributed to activation of the Hh pathway. A greater understanding of the role of the Hh signaling pathway in TNBC may lead to an effective strategy for TNBC prevention by developing molecular tools that interfere with it.

\section{Acknowledgements}

The authors thank Bo Wang and Junjun Zhao for the skillful technical assistance. This study was supported by The Ministry of Education Doctor Special Fund in Colleges and Universities of China (No. 200801610001), and the National Natural Science Fund of China (No. 30870968). Y.T. planned, set up the experiments, collected the data, analyzed and interpreted the results and drafted the manuscript. J.M. collected the clinical specimens and patient data. Q.Z. participated in the immunoassays. L.L. planned, supervised and provided financial and technical support for the study. All authors have read and approved the final manuscript.

\section{References}

1. Köster F, Engel JB, Schally AV, et al: Triple-negative breast cancers express receptors for growth hormone-releasing hormone (GHRH) and respond to GHRH antagonists with growth inhibition. Breast Cancer Res Treat 116: 273-279, 2009.

2. Carey LA, Dees EC, Sawyer L, et al: The triple negative paradox: primary tumor chemosensitivity of breast cancer subtypes. Clin Cancer Res 13: 2329-2334, 2007.

3. Sotiriou C, Phil D and Pusztaj J: Gene-expression signatures in breast cancer. N Engl J Med 360: 790-800, 2009.

4. Liedtke C, Mazouni C, Hess KR, et al: Response to neoadjuvant therapy and long-term survival in patients with triple-negative breast cancer. J Clin Oncol 26: 1275-1281, 2008.

5. De Ruijter TC, Veeck J, de Hoon JPJ, van Engeland M and Tjan-Heijnen VC: Characteristics of triple-negative breast cancer. J Cancer Res Clin Oncol 137: 183-192, 2011.

6. Rakha EA, Elsheikh SE, Aleskandarany MA, et al: Triplenegative breast cancer: distinguishing between basal and nonbasal subtypes. Clin Cancer Res 15: 2302-2310, 2009.

7. Lim KH, Oh DY, Chie EY, et al: Metaplastic breast carcinoma: clinicopathologic features and prognostic value of triple negativity. Jpn J Clin Oncol 40: 112-118, 2010

8. Tischkowitz M, Brunet JS, Bégin LR, Huntsman DG, Cheang MC, Akslen LA, Nielsen TO and Foulkes WD: Use of immunohistochemical markers can refine prognosis in triple negative breast cancer. BMC Cancer 7: 134, 2007.

9. Lin NU, Claus E, Sohl J, Razzak AR, Arnaout A and Winer EP: Sites of distant relapse and clinical outcomes in patients with metastatic triple-negative breast cancer: high incidence of central nervous system metastases. Cancer 113: 2638-2645, 2008.

10. Chacón RD and Costanzo MV: Triple-negative breast cancer. Breast Cancer Res 12 (Suppl 2): 3, 2010.

11. Ross JS, Fletcher JA, Bloom KJ, Linette GP, Stec J, Symmans WF, Pusztai L and Hortobagyi GN: Targeted therapy in breast cancer:the HER-2/neu gene and protein. Mol Cell Proteomics 3: 379-398, 2004.

12. Zhang XM, Harrington N, Moraes RC, Wu MF, Hilsenbeck SG and Lewis MT: Cyclopamine inhibition of human breast cancer cell growth independent of Smoothened (Smo). Breast Cancer Res Treat 115: 505-521, 2009. 
13. Oro AE, Higgins KM, Hu Z, Bonifas JM, Epstein EH Jr and Scott MP: Basal cell carcinomas in mice overexpressing sonic Hedgehog. Science 276: 817-821, 1997.

14. Han ME, Lee YS, Baek SY, Kim BS, Kim JB and Oh SO Hedgehog signaling regulates the survival of gastric cancer cells by regulating the expression of Bcl-2. Int J Mol Sci 10: 3033-3043, 2009.

15. Dormoy V, Danilin S, Lindner V, et al: The sonic hedgehog signaling pathway is reactivated in human renal cell carcinoma and plays orchestral role in tumor growth. Mol Cancer 8: 123, 2009.

16. Chen M, Tanner M, Levine AC, Levina E, Ohouo $P$ and Buttyan R: Androgenic regulation of hedgehog signaling pathway components in prostate cancer cells. Cell Cycle 8: 149-157, 2009.

17. Liu S, Dontu G and Wicha MS: Mammary stem cells, selfrenewal pathways, and carcinogenesis. Breast Cancer Res 7 : $86-95,2005$.

18. Zhou JX, Jia LW, Liu WM, Miao CL, Liu S, Cao YJ and Duan EK: Role of sonic hedgehog in maintaining a pool of proliferating stem cells in the human fetal epidermis. Hum Reprod 21: 1698-1704, 2006.

19. Kasper M, Jaks V, Fiaschi M and Toftgård R: Hedgehog signaling in breast cancer. Carcinogenesis 30: 903-911, 2009.

20. Liu S, Dontu G, Mantle ID, Patel S, Ahn NS, Jackson KW, Suri $\mathrm{P}$ and Wicha MS: Hedgehog signaling and Bmi-1 regulate self-renewal of normal and malignant human mammary stem cells. Cancer Res 66: 6063-6071, 2006.

21. Singletary SE and Connolly JL: Breast cancer staging: working with the sixth edition of the AJCC Cancer Staging Manual. CA Cancer J Clin 56: 37-47, 2006.

22. Sinicrope FA, Ruan SB, Cleary KB, Stephens LC, Lee JJ and Levin B: bcl-2 and p53 oncoprotein expression during colorectal tumorigenesis. Cancer Res 55: 237-241, 1995.

23. Ten Haaf A, Bektas N, von Serenyi S, Losen I, Arweiler EC, Hartmann A, Knüchel R and Dahl E: Expression of the gliomaassociated oncogene homolog (GLI) 1 in human breast cancer is associated with unfavourable overall survival. BMC Cancer 9: 298-309, 2009
24. Pan S, Dong Q, Sun LS and Li TJ: Mechanisms of inactivation of PTCH1 gene in nevoid basal cell carcinoma syndrome: modification of the two-hit hypothesis. Clin Cancer Res 16: 442-450, 2010.

25. Yauch RL, Dijkgraaf GJ, Alicke B, et al: Smoothened mutation confers resistance to a Hedgehog pathway inhibitor in medulloblastoma. Science 326: 572-574, 2009.

26. Epstein EH: Basal cell carcinomas: attack of the hedgehog. Nat Rev Cancer 8: 743-754, 2008.

27. Peacock CD, Wang Q, Gesell GS, et al: Hedgehog signaling maintains a tumor stem cell compartment in multiple myeloma. Proc Natl Acad Sci USA 104: 4048-4053, 2007.

28. Moraes RC, Zhang $\mathrm{X}$, Harrington N, Fung JY, Wu MF, Hilsenbeck SG, Allred DC and Lewis MT: Constitutive activation of smoothened (SMO) in mammary glands of transgenic mice leads to increased proliferation, altered differentiation and ductal dysplasia. Development 134: 12311242,2007

29. Gao J, Graves S, Koch U, Liu S, et al: Hedgehog signaling is dispensable for adult hematopoietic stem cell function. Cell Stem Cell 4: 548-558, 2009.

30. Zhao C, Chen A, Jamieson $\mathrm{CH}$, et al: Hedgehog signaling is essential for maintenance of cancer stem cells in myeloid leukaemia. Nature 458: 776-779, 2009.

31. Zardawi SJ, O'Toole SA, Sutherland RL and Musgrove EA: Dysregulation of Hedgehog, Wnt and Notch signaling pathways in breast cancer. Histol Histopathol 24: 385-398, 2009.

32. Zhao J, Chen G, Cao D, Li Y, Diao F, Cai H, Jin Y and Lu J: Expression of Gli1 correlates with the transition of breast cancer cells to estrogen-independent growth. Breast Cancer Res Treat 119: 39-51, 2010.

33. Boman BM and Wicha MS: Cancer stem cells: a step toward the cure. J Clin Oncol 26: 2795-2799, 2008 